\title{
PERFIL EPIDEMIOLÓGICO DE PACIENTES COM LEISHMANIOSE VISCERAL NAS REGIÕES DO BRASIL
}

\author{
Erilana Silva Pacheco ${ }^{1}$ \\ Josiane Silva de Lima Azevedo ${ }^{2}$ \\ Yrlane Paes Ferreira ${ }^{3}$ \\ Sylvia de Fátima dos Santos Guerra ${ }^{4}$ \\ Márcia Cristina dos Santos Guerra ${ }^{5}$
}

\begin{abstract}
RESUMO
A Leishmaniose visceral (LV) é uma doença endêmica no Brasil que pode ocasionar óbito do indivíduo em 95\% dos casos não tratados, sendo considerada um problema de saúde pública. O presente estudo teve como objetivo descrever o perfil epidemiológico da LV das diferentes regiões do Brasil. Para isso, os dados analisados das regiões Norte, Nordeste, Sul, Sudeste e Centro-Oeste do Brasil no período de 2014 a 2018 foram coletados do Sistema de Informação de Agravos de Notificação, sendo analisada a incidência, letalidade, gênero, faixa etária, escolaridade, critério de confirmação e evolução do caso empregando o teste de Qui-quadrado, teste $\mathrm{G}$ e regressão linear simples. Durante o período do estudo ocorreram 19.053 casos de LV no Brasil, com maior incidência nas regiões Norte e Nordeste. Ocorrência maior na faixa etária menor de 14 anos, baixa escolaridade, gênero masculino, que obtiveram diagnóstico laboratorial e cura. Casos de LV ainda são recorrentes no cenário atual, sendo necessário ações efetivas nos diferentes níveis de prevenção podem reduzir significativamente os índices desta doença no Brasil.
\end{abstract}

Palavras-chave: Leishmaniose Visceral Humana. Perfil epidemiológico. Leshmaniose.

\section{EPIDEMIOLOGICAL PROFILE OF PATIENTS WITH VISCERAL LEISHMANIASIS IN BRAZILIAN REGIONS}

\begin{abstract}
Visceral Leishmaniasis (VL) is an endemic disease in Brazil and can cause death in 95\% of untreated cases, being considered a public health problem. The present study aimed to describe the epidemiological profile of VL in different regions of Brazil. For this, the analyzed data from the North, Northeast, South, Southeast and Midwest regions of Brazil in the period from 2014 to 2018 were collected from the Notifiable Diseases Information System, analyzing the incidence, lethality, gender, age group, schooling, confirmation criteria and case evolution using the Chi-square test, $\mathrm{G}$ test and simple linear regression. During the study period, there were 19,053 cases of VL in Brazil, with a higher incidence in the North and Northeast regions. Most occurrence in the age group under 14 years, low education, male, who obtained laboratory diagnosis and cure. VL cases are still recurrent in the current scenario, requiring effective actions at different levels of prevention can significantly reduce the rates of this disease in Brazil.
\end{abstract}

${ }^{1}$ Graduada no curso de Bacharelado em Biomedicina do Centro Universitário Metropolitano da Amazônia (UNIFAMAZ). E-mail: erilanapacheco0712@gmail.com.

${ }^{2}$ Graduada no curso de Bacharelado em Biomedicina do Centro Universitário Metropolitano da Amazônia (UNIFAMAZ). E-mail: josianeazevedo.ufra@gmail.com.

${ }^{3}$ Graduada no curso de Bacharelado em Biomedicina do Centro Universitário Metropolitano da Amazônia (UNIFAMAZ). E-mail: yrlanebiomedicina@gmail.com.

${ }^{4}$ Doutora em Doenças Tropicais. Docente do Centro Universitário Metropolitano da Amazônia (UNIFAMAZ); Pesquisadora colaboradora do Instituto Evandro Chagas (IEC). E-mail: sylviafsguerra @ gmail.com.

${ }^{5}$ Mestre em Saúde, Sociedade e Endemias na Amazônia pela Universidade Federal do Pará (UFPa). Docente do Centro Universitário Metropolitano da Amazônia (UNIFAMAZ). E-mail: marciacsguerra@ gmail.com. 
Keywords: Human Visceral Leishmaniasis. Epidemiological profile. Leishmaniasis.

Data de submissão: 25.04 .2021

Data de aprovação: 16.05 .2021

\section{INTRODUÇÃOO}

Agentes etiológicos como os protozoários, bactérias, vírus e fungos são responsáveis pelas doenças infectocontagiosas, que se caracterizam pelo desequilíbrio entre a relação parasita-hospedeiro ${ }^{[1,2]}$. Tal relação é formada por uma tríade: a suscetibilidade do hospedeiro, infecciosidade/virulência do parasita e as condições ambientais, o desequilíbrio desses sistemas resulta no aparecimento de uma doença específica, todavia, uma quarta variável pode estar envolvida neste processo que são os vetores, considerados como transmissores de doenças ${ }^{[3,4]}$.

Segundo a Agência Nacional de Águas (ANA), aproximadamente $45 \%$ da população brasileira possui tratamento inadequado de esgoto e grande parte dos domicílios destina seu esgoto sanitário diretamente em corpos de água, essa falta de condições higiênico-sanitárias adequadas, gera o acometimento dessa população por diversas doenças causadas por patógenos de veiculação hídrica, bem como serve de criadouro para artrópodes vetores de diversas doenças ${ }^{[5-7]}$. Observando o cenário dos últimos 50 anos no Brasil, doenças transmitidas por vetores e as relacionadas a saneamento básico são as mais comuns em nosso país, dentre eles podemos citar: ascaridíase, amebíase, giardíase, toxoplasmose, doenças de chagas, malária e leishmaniose ${ }^{[1,8]}$.

A Leishmaniose é uma doença causada pelo protozoário da família Trypanosomatidae e gênero Leishmania com mais de 20 espécies, onde tais espécies são transmitidas principalmente através da picada de flebotomíneos fêmeas infectadas, pois as mesmas, tendem a se alimentar de sangue para obter proteínas e aminoácidos para o desenvolvimento dos ovos, enquanto que, os flebotomíneos machos não são hematófagos. Os animais selvagens e domésticos costumam ser o principal reservatório do gênero Leishmania, no qual, o reservatório tende a variar de acordo com a espécie ${ }^{[9-10]}$. Tal doença é dividida em três formas: cutânea (mais comum), mucocutânea e a visceral (forma mais grave) ${ }^{[9-10]}$.

A Leishmaniose visceral (LV) é uma forma grave que pode ocasionar o óbito do indivíduo em $95 \%$ dos casos não tratados. Segundo a OMS, estima-se que ocorra anualmente em todo o mundo de 50 mil a 90 mil casos novos, sendo a maioria não notificado. Em 2018, observou-se que $95 \%$ dos casos notificados concentravam-se em apenas 10 países, estando o Brasil incluso nesta realidade. A LV pode apresentar como manifestação clínica febre alta, perda de peso, palidez nas mucosas, hepatoesplenomegalia discreta, por vezes, tosse e diarreia podendo a sintomatologia agravar com o emagrecimento progressivo levando o paciente a desnutrição e caquexia acentuada, sendo comum nesses casos o edema generalizado, dispneia, dores musculares, problemas digestivos e epistaxe. ${ }^{[9-10]}$

Esta doença frequentemente acomete indivíduos em baixas condições socioeconômicas, estando relacionados à desnutrição, condições inadequadas de moradia e saneamento, sistema imunológico debilitado e carência de recursos financeiros. Características do local de residência, tal como alterações climáticas, tornam áreas endêmicas para Leishmaniose. ${ }^{[9-12]}$

Conhecendo as regiões e a população mais suscetíveis ao desenvolvimento da doença, ações de prevenção em saúde podem ser desenvolvidas visando reduzir o impacto do agravo na saúde pública e restaurando a qualidade de vida da população. Desta forma, o presente estudo tem como objetivo descrever os aspectos epidemiológicos da LV nas diferentes regiões do Brasil. 


\section{MÉTODOS}

Trata-se de um estudo epidemiológico descritivo de série histórica, sobre casos de LV nas regiões (Norte, Nordeste, Sudeste, Sul e Centro-oeste) do Brasil, no período de 2014 a 2018, na qual os dados dos casos confirmados foram obtidos através do Sistema de Informação de Agravos de Notificação (SINANNET), disponível no site do Departamento de Informática do Sistema Único de Saúde (DATASUS) ${ }^{[13]}$ sendo acessado pelo endereço eletrônico: http://www.datasus.gov.br.

A obtenção dos dados foi feita através do DATASUS durante o período de julho/2020 a agosto/2020. Na plataforma, foram selecionadas todas as regiões do Brasil, categorizadas pelas seguintes variáveis representadas na Tabela 1.

As características gerais dos casos com LV foram descritas usando a estatística descritiva (as variáveis categóricas foram apresentadas em frequências e percentuais e as variáveis numéricas em mediana e desvio-quartílico). Para comparação das macrorregiões em relação a variável sexo, idade, escolaridade, critério de confirmação diagnóstica e evolução do caso foi utilizado o teste de Qui-quadrado e teste G. Foi utilizado a regressão linear simples para avaliar a tendência da taxa de incidência e taxa de mortalidade nas macrorregiões. As análises estatísticas foram calculadas usando o programa Bioesta 5.3. O nível de significância adotado foi de $5 \%$.

Tabela 1- Categorização das variáveis independentes para o cálculo das razões de incidência

\begin{tabular}{ccc}
\hline Nome da variável & Nível & Significado \\
\hline Sexo & 1 & Masculino \\
& 2 & Feminino \\
Grupos de idades & 1 & $<14$ anos \\
& 2 & $15-19$ anos \\
Escolaridade & 3 & $20-39$ anos \\
& 4 & $40-59$ anos \\
& 5 & $>=60$ anos \\
Critério de & 1 & Analfabeto \\
Confirmação & 2 & Ensino Fund. \\
& 3 & Completo \\
& 4 & Ensino Médio \\
Evolução do Caso & 1 & Completo \\
& 2 & Ensino Superior \\
& 1 & Laboratorial \\
& 2 & Clínico - \\
& 3 & Epidemiológico \\
& 4 & Cura da LV \\
& 5 & Abandono da LV \\
& & Óbito por LV \\
& & Óbito por outra \\
& & causa \\
& & Transferência \\
\hline
\end{tabular}

Fonte: Autoria Própria.

Foram utilizados dados de estimativas populacionais das regiões do Brasil para o cálculo da taxa de 100.000 habitantes nos anos de 2014 a 2018, coletados no site do Instituto Brasileiro de Geografia e Estatística (IBGE) ${ }^{[14]}$, acessado pelo endereço eletrônico: 
https://www.ibge.gov.br/estatisticas/sociais/populacao/9103-estimativas-de-populacao.

\section{RESULTADOS}

Com base nos dados coletados, estima-se que durante o período do estudo, o Brasil tenha aproximadamente 19.053 casos em todas as suas regiões, demonstrando um percentual de $19,05 \%$ em relação ao número de habitantes.

Ao observar a incidência por região, conforme exposto na Figura 1, nota-se um maior número de casos nas regiões Norte e Nordeste no período de 2014 a 2018.

Figura 1 - Incidência de casos de LV notificados por região - 2014 a 2018

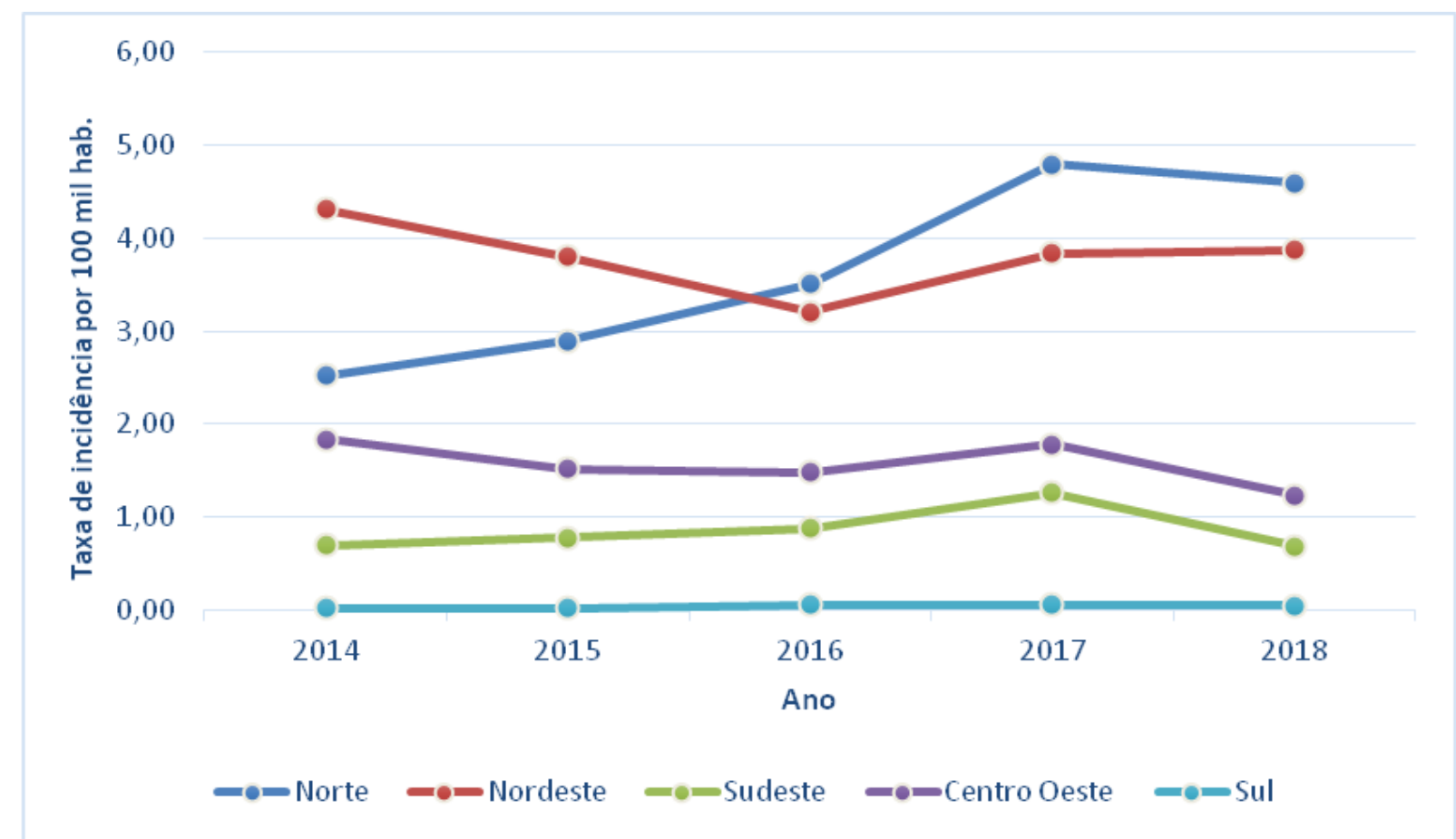

Fonte: Ministério da Saúde/SVS-Sistema de Informação de agravos de Notificação-

SinanNet,Agosto/2020.

O dado referente ao aumento na taxa de incidência de LV em grandes regiões do Brasil no período estudado mostra tendência crescente na região Norte, a cada ano, em média, há um aumento de 0,61 por 100.000 habitantes. As outras regiões apresentaram estabilidade na taxa de incidência (Tabela 2).

Tabela 2 - Tendência da taxa de incidência de LV por regiões do Brasil- 2014 a 2018

\begin{tabular}{ccccccccc}
\hline Ano & $\mathbf{2 0 1 4}$ & $\mathbf{2 0 1 5}$ & $\mathbf{2 0 1 6}$ & $\mathbf{2 0 1 7}$ & $\mathbf{2 0 1 8}$ & $\begin{array}{c}\text { Equação de } \\
\text { Regressão }\end{array}$ & $\begin{array}{c}\text { Coef. } \\
\text { Determinação }\end{array}$ & $\begin{array}{c}\text { Valor } \\
\text { de p }\end{array}$ \\
\hline Norte & 2.52 & 2.90 & 3.51 & 4.80 & 4.60 & $\mathrm{y}=0.61 \mathrm{x}+1.85$ & $\mathrm{R}^{2}=0.90$ & 0.0123 \\
Nordeste & 4.31 & 3.80 & 3.21 & 3.84 & 3.87 & $\mathrm{y}=-0.08 \mathrm{x}+4.06$ & $\mathrm{R}^{2}=0.11$ & 0.5787 \\
Sudeste & 0.69 & 0.77 & 0.88 & 1.26 & 0.69 & $\mathrm{y}=0.05 \mathrm{x}+0.72$ & $\mathrm{R}^{2}=0.10$ & 0.5942 \\
Centro Oeste & 1.84 & 1.52 & 1.48 & 1.78 & 1.24 & $\mathrm{y}=-0.09 \mathrm{x}+1.86$ & $\mathrm{R}^{2}=0.37$ & 0.2734 \\
Sul & 0.02 & 0.02 & 0.06 & 0.06 & 0.05 & $\mathrm{y}=0.01 \mathrm{x}+0.01$ & $\mathrm{R}^{2}=0.63$ & 0.1259 \\
\hline
\end{tabular}

Fonte: Autoria Própria.

$\mathrm{Na}$ associação das taxas de incidência das variáveis por macrorregiões, o sexo 
masculino apresentou predomínio em todas as regiões, com diferença significativa $(p=0,002)$ entre as regiões.

A variável faixa etária apresentou diferença significativa $(p=0,0001)$ entre as regiões onde, a maioria dos casos estão concentrados na faixa etária $\geq 14$ anos nas regiões Norte, Nordeste, Sudeste e Centro-Oeste, exceto na região Sul, na qual o número de casos foi maior entre 20-39 anos.

O grau de escolaridade apresentou significância ( $p<0,0001)$, onde o nível analfabeto e fundamental completo apresentou-se elevado nas regiões do Brasil.

$\mathrm{Na}$ variável evolução do caso e critério de confirmação também se observou significância estatística ( $p<0,0001)$, tendo a região Nordeste com maior frenquência de cura e a região Centro - Oeste com menor frequência, no critério abandono as regiões Sudeste e Sul apresentaram menores frequências, enquanto que a região Norte teve maior frequência de casos, com taxa menor de óbitos na região Sul.

Em relação aos critérios laboratoriais, a região Nordeste apresentou baixa frequência de casos, enquanto que a região Sudeste notificou alta frequência. Na escala critério clínico, a região Nordeste apresentou alta frequência, as regiões Norte, Sudeste e Centro-Oeste mostraram estabilidade e a região sul apresentou baixa frequência (Tabela 3).

Tabela 3 - Taxa de incidência das variáveis por regiões, 2014 a 2018

\begin{tabular}{|c|c|c|c|c|c|c|c|c|c|c|c|c|c|}
\hline & $\begin{array}{l}\text { Norte } \\
n=329\end{array}$ & $\%$ & $\begin{array}{l}\text { Nordeste } \\
n=10794\end{array}$ & $\%$ & $\begin{array}{l}\text { Sudeste } \\
n=3711\end{array}$ & $\%$ & $\begin{array}{c}\text { Centro- } \\
\text { 0este } \\
n=1228 \\
\end{array}$ & $\%$ & $\begin{array}{c}\text { Sul } \\
n=61\end{array}$ & $\%$ & $\begin{array}{c}\text { Total } \\
\mathbf{n}=\mathbf{1 9 0 5 3}\end{array}$ & $\%$ & $\begin{array}{l}\text { Valor } \\
\text { de p }\end{array}$ \\
\hline \multicolumn{14}{|l|}{ Gênero } \\
\hline Masculino & 2049 & 62.9 & 7199 & 66.7 & 2422 & 65.3 & 808 & 65.8 & 42 & 68.9 & 12520 & 65.7 & $0.002^{\mathrm{a}}$ \\
\hline Feminino & 1210 & 37.1 & 3595 & 33.3 & 1289 & 34.7 & 420 & 34.2 & 19 & 31.1 & 6533 & 34.3 & \\
\hline \multicolumn{14}{|l|}{ Faixa etária } \\
\hline$<14$ anos & 1782 & 54.7 & 5064 & 46.9 & 1282 & 34.5 & 412 & 33.6 & 17 & 27.9 & 8557 & 44.9 & $<0.0001^{\mathrm{b}}$ \\
\hline 15 - 19 ANOS & 173 & 5.3 & 571 & 5.3 & 150 & 4.0 & 45 & 3.7 & 2 & 3.3 & 941 & 4.9 & \\
\hline 20-39 ANOS & 715 & 21.9 & 2514 & 23.3 & 840 & 22.6 & 317 & 25.8 & 18 & 29.5 & 4404 & 23.1 & \\
\hline 40-59 ANOS & 418 & 12.8 & 1879 & 17.4 & 916 & 24.7 & 288 & 23.5 & 11 & 18.0 & 3512 & 18.4 & \\
\hline$>=60$ & 171 & 5.2 & 765 & 7.1 & 523 & 14.1 & 166 & 13.5 & 13 & 21.3 & 1638 & 8.6 & \\
\hline EM BRANCO/Ign & 0 & 0.0 & 1 & 0.0 & 0 & 0.0 & 0 & 0.0 & 0 & 0.0 & 1 & 0.0 & \\
\hline \multicolumn{14}{|l|}{ Escolaridade } \\
\hline ANALFABETO & 80 & 2.5 & 431 & 4.0 & 84 & 2.3 & 22 & 1.8 & 0 & 0.0 & 617 & 3.2 & $<0.0001^{\mathrm{b}}$ \\
\hline Fundamental & 892 & 27.4 & 3124 & 28.9 & 1026 & 27.6 & 325 & 26.5 & 15 & 24.6 & 5382 & 28.2 & \\
\hline Médio & 302 & 9.3 & 867 & 8.0 & 333 & 9.0 & 145 & 11.8 & 10 & 16.4 & 1657 & 8.7 & \\
\hline Superior & 40 & 1.2 & 67 & 0.6 & 47 & 1.3 & 34 & 2.8 & 2 & 3.3 & 190 & 1.0 & \\
\hline IGN/BRANCO* & 504 & 15.5 & 2184 & 20.2 & 1184 & 31.9 & 340 & 27.7 & 19 & 31.1 & 4231 & 22.2 & \\
\hline NÃO SE APLICA** & 1441 & 44.2 & 4121 & 38.2 & 1037 & 27.9 & 362 & 29.5 & 15 & 24.6 & 6976 & 36.6 & \\
\hline \multicolumn{14}{|l|}{ Evolução do caso } \\
\hline Cura & 2428 & 74.5 & 6825 & 63.2 & 2929 & 78.9 & 37 & 3.0 & 37 & 60.7 & 12256 & 64.3 & $<0.0001^{\mathrm{b}}$ \\
\hline Abandono & 31 & 1.0 & 83 & 0.8 & 16 & 0.4 & 12 & 1.0 & 1 & 1.6 & 143 & 0.8 & \\
\hline Óbito & 164 & 5.0 & 812 & 7.5 & 358 & 9.6 & 90 & 7.3 & 12 & 19.7 & 1436 & 7.5 & \\
\hline Óbito por outra causa & 85 & 2.6 & 223 & 2.1 & 109 & 2.9 & 63 & 5.1 & 2 & 3.3 & 482 & 2.5 & \\
\hline Transferência* & 127 & 3.9 & 920 & 8.5 & 64 & 1.7 & 35 & 2.9 & 1 & 1.6 & 1147 & 6.0 & \\
\hline Ign\branco* & 424 & 13.0 & 1931 & 17.9 & 235 & 6.3 & 101 & 8.2 & 8 & 13.1 & 2699 & 14.2 & \\
\hline \multicolumn{14}{|l|}{ Critério de confirmação } \\
\hline Laboratorial & 2926 & 89.8 & 8947 & 82.9 & 3453 & 93.0 & 1101 & 89.7 & 51 & 83.6 & 16478 & 86.5 & $<0.0001^{\mathrm{a}}$ \\
\hline Clínico epidemiológico & 333 & 10.2 & 1847 & 17.1 & 258 & 7.0 & 127 & 10.3 & 10 & 16.4 & 2575 & 13.5 & \\
\hline
\end{tabular}

${ }^{\text {a }}$ Teste do qui-quadrado 
${ }^{\mathrm{b}}$ Teste $\mathrm{G}$

Fonte: Autoria Própria.

A taxa de mortalidade apresentou tendência crescente na região Norte, a cada ano, em média há um aumento na taxa de mortalidade de 0,05 por 100.000 habitantes. As outras regiões apresentaram estabilidade na taxa (Tabela 4 ).

Tabela 4 - Taxa de mortalidade de LV por regiões do Brasil- 2014 a 2018

\begin{tabular}{lcccccccc}
\hline & $\mathbf{2 0 1 4}$ & $\mathbf{2 0 1 5}$ & $\mathbf{2 0 1 6}$ & $\mathbf{2 0 1 7}$ & $\mathbf{2 0 1 8}$ & $\begin{array}{c}\text { Equação de } \\
\text { regressão }\end{array}$ & $\begin{array}{c}\text { Coef. } \\
\text { Determinação }\end{array}$ & $\begin{array}{c}\text { Valor } \\
\text { de p }\end{array}$ \\
\hline Norte & & & & & & & & \\
Nordeste & 0.093 & 0.177 & 0.136 & 0.190 & 0.324 & $\mathrm{y}=0.05 \mathrm{x}+0.04$ & $\mathrm{R}^{2}=0.74$ & 0.0580 \\
Sudeste & 0.274 & 0.293 & 0.269 & 0.304 & 0.291 & $\mathrm{y}=0.004 \mathrm{x}+0.27$ & $\mathrm{R}^{2}=0.23$ & 0.6002 \\
Centro Oeste & 0.065 & 0.070 & 0.086 & 0.129 & 0.065 & $\mathrm{y}=0.01 \mathrm{x}+0.06$ & $\mathrm{R}^{2}=0.12$ & 0.5727 \\
Sul & 0.145 & 0.149 & 0.096 & 0.088 & 0.099 & $\mathrm{y}=-0.02 \mathrm{x}+0.16$ & $\mathrm{R}^{2}=0.68$ & 0.0812 \\
& 0.003 & 0.000 & 0.020 & 0.013 & 0.003 & $\mathrm{y}=0.001 \mathrm{x}+0.004$ & $\mathrm{R}^{2}=0.06$ & 0.6900 \\
\hline
\end{tabular}

Fonte: Autoria Própria.

A taxa de letalidade nas macrorregiões apresentou estabilidade em praticamente todas as regiões, com exceção da região Sul que apresentou altas taxas de letalidade, no ano de 2016 e posterior queda nos anos de 2017 e 2018, conforme demonstrado na Figura 2.

Figura 2 - Taxa de Letalidade de LV notificados por região - 2014 a 2018

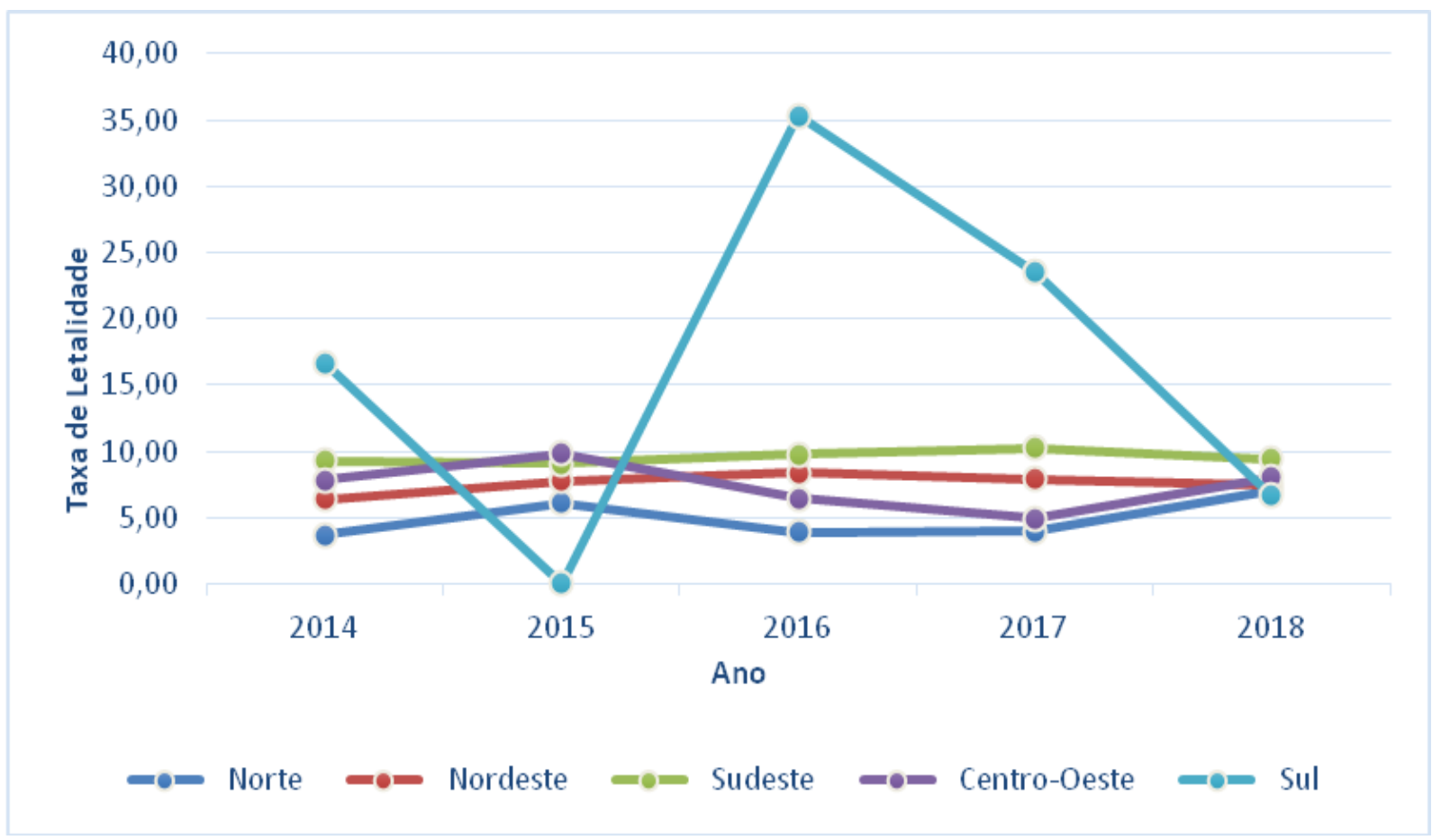

Fonte: Ministério da Saúde/SVS-Sistema de Informação de agravos de Notificação-Sinan Net,Agosto/2020.

\section{DISCUSSÃO}

Com a diminuição das áreas de florestas ocorre o aumento da extensão demográfica 
facilitando a entrada da Leishmaniose nos centros. Durante o estudo foram obtidos dados referentes ao perfil epidemiológico de pacientes com LV nas diferentes regiões do Brasil.

A incidência média da LV nas regiões do Brasil oscilou durante o período estudado, com maior detecção nas regiões Norte e Nordeste. Tais dados do Nordeste também foram relatados em estudo conduzido por Lucena; Medeiros ${ }^{[15]}$, analisando casos novos dos diferentes estados da região Nordeste no período de 2010 a 2017, relataram variação da incidência e declínio na região e, apesar da diminuição dos casos, o Nordeste apresenta uma das maiores incidências média de LV do Brasil.

Segundo Barbosa; Guimarães; Luz ${ }^{[16]}$, as populações mais vulneráveis estão nas áreas periféricas, onde não possuem um saneamento básico, realidade observada na região Norte e Nordeste, as quais apresentam as mais precárias condições sanitárias do Brasil ${ }^{[17]}$.

A faixa etária mais acometida por LV foi $<14$ anos, o que corrobora com dados expostos em estudo conduzido por Farias et al ${ }^{[18]}$ realizado na região Norte de Minas Gerais, no período de 2011 a 2015 e por Barbosa ${ }^{[19]}$ em Rio Grande do Norte, no período de 2007 a 2015. Possivelmente, tal fato é associado a imaturidade imunológica desta faixa etária ${ }^{[19,20]}$.

No presente estudo pode ser observado que o sexo masculino foi o mais acometido pelo LV. Tal realidade também foi relatada em diferentes estudos tal como Barbosa ${ }^{[19]}$, Farias et $a l^{[18]}$, Rocha et $a l^{[21]}$ e Ortiz; Anversa ${ }^{[22]}$, estando possivelmente associado a uma maior exposição ao vetor devido atividades laborais.

Comparando o presente estudo com o citado, conclui-se que, mesmo essas regiões apresentando redução na taxa de incidência, ainda assim, a região Nordeste permanece sendo a região com a maior prevalência da doença em relação as demais regiões do Brasil.

Em relação ao nível de escolaridade, a predominância dos casos se dá nas classes, analfabeto e ensino fundamental em todas as regiões do Brasil, descritas, de acordo com dados colhidos para o estudo em questão, sendo tais dados observados em estudo realizado em Fortaleza ${ }^{[23]}$ no período de 2007 a 2017 e Pernambuco entre os anos de 2003 e $2015^{[24]}$. Como afirmado por Almeida et al ${ }^{[23]}$, a baixa escolaridade pode sugerir que estão mais vulneráveis a doença pessoas com baixas condições socioeconômicas.

A maioria dos casos evoluiu a cura e obteve diagnóstico laboratorial, situação também observada por Almeida et al ${ }^{[23]} \mathrm{em}$ Fortaleza, o que demonstra possivelmente que quando há confirmação da doença logo o tratamento é implementado e a saúde dos acometidos restaurada.

A taxa de mortalidade que apresentou tendência crescente foi a região Norte, a qual apresentou uma das maiores incidências da doença, enquanto que a letalidade demonstrou não variar no tempo estudado, com exceção da região sul que apresentou um pico no ano de 2016. Tal região foi a última no Brasil a notificar casos de $\mathrm{LV}^{[25]}$.

Desta forma, observa-se que a LV ainda é uma realidade no Brasil, acometendo principalmente indivíduos com baixas condições socioeconômicas. Há programas de saúde que desenvolvem ações de controle estando focados em detectar, diagnosticar e tratar os casos notificados. No entanto, mesmo com um amplo programa de controle nas regiões, a LV ainda é um problema de saúde pública e ações atuantes nos diferentes níveis de prevenção podem reduzir significativamente os índices desta doença.

Para tanto, se faz necessário a busca incessante por estratégias que auxiliem no planejamento de novas metodologias para ações voltadas ao combate de vetores e reservatórios, com alternativas precisas de prevenção, diagnóstico, monitoramento, tratamento, e cuidados da popopulação, bem como, políticas de saúde pública para serem aplicadas nessas regiões, resultando na diminuição do número de casos e notificações nas regiões do Brasil. 


\section{REFERÊNCIAS}

ABES. Associação Brasileira de Engenharia Sanitária e Ambiental. Situação do Saneamento Básico no Brasil: Uma Análise Com Base na PNAD 2015, 2015. http://abesdn.org.br/pdf/Situacao.pdf

ALMEIDA, Clarice Pessoa et al. Leishmaniose visceral: distribuição temporal e espacial em Fortaleza, Ceará, 2007-2017. Epidemol Serv Saúde [preprint]. 2020.

BARBOSA, Isabelle Ribeiro. Leishmaniose Visceral Humana no Município de Natal-RN: Análise Clínico-Epidemiológica e Espacial. Revista Ciência Plural. v. 2, n. 1, p:89-101, 2016. https://periodicos.ufrn.br/rcp/article/view/8559/7120

BARBOSA, Miriam Nogueira; GUIMARÃES, Eliete Alabano de Azevedo; LUZ, Zélia Maria Profeta da. Avaliação de estratégia de organização de serviços de saúde para prevenção e controle da leishmaniose visceral. Epidemiologia e Serviços de Saúde. v. 25, n. 3, p. 563574, jul/set. 2016.

BRASIL. Agência Nacional de Água (ANA) Atlas esgotos: despoluição de bacias hidrográficas. Brasília: Agência Nacional de Águas, Secretaria Nacional de Saneamento Ambiental, 2017. Disponível em: https://www.ana.gov.br/noticias/atlas-esgotos-revela-maisde-110-mil-km-de-rios-com-comprometimento-da-qualidade-da-agua-por-cargaorganica/atlaseesgotosdespoluicaodebaciashidrograficas-resumoexecutivo_livro.pdf/view. Acesso em: 20 jan. 2021.

BRASIL. Ministério da Saúde. Guia de Vigilância em Saúde. 3 ed. Volume único. 2019.

BRASIL. Ministério do Planejamento, Orçamento e Gestão. Instituto Brasileiro de Geografia e Estatística (IBGE). Panorama do Brasil, 2016. Disponível em: https://cidades.ibge.gov.br/brasil/panorama. Acesso em: 20 jan. 2021.

BRASIL. Ministério do Planejamento, Orçamento e Gestão. Instituto Brasileiro de Geografia e Estatística (IBGE). Estimativas da População. Disponível em: https://www.ibge.gov.br/estatisticas/sociais/populacao/9103-estimativas-de-populacao.

DATASUS - Departamento de Informática do SUS. Indicadores e dados básicos, 2013 [citado em 14 de maio de 2020]. Disponível em:http://tabnet.datasus.gov.br/cgi/idb2013/matriz.htm.

DIAS-LIMA, Artur. Ecologia médica: uma visão holística no contexto das enfermidades humanas. Revista Brasileira de Educação Médica. v. 38, n. 2, p. 165-172, abr/jun. 2014.

FARIAS, Hildeth Maísa Torres et al. Perfil epidemiológico da leishmaniose visceral humana nas regiões de saúde do norte de minas gerais. Enferm. Foco. v. 10, n. 2, p. 90-96, 2019. http://revista.cofen.gov.br/index.php/enfermagem/article/view/1887/526.

LEMOS, Maria Deuzina Alves; SOUSA Odaíris Holanda de; SILVA, Zilene do Socorro Santa Brígida. Perfil da Leishmaniose visceral no Brasil: uma revisão bibliográfica. J Business Techn. v. 9, n. 1, p:93-114, 2019. https://jnt1.websiteseguro.com/index.php/JNT/article/viewFile/399/328. 
LISBOA, Antonio Ricardo et al. Leishmaniose visceral: Uma revisão literária. Revista Brasileira de Educação e Saúde. v. 6, n. 2, p. 35-43, abr/jun. 2016.

LUCENA, Robespierre Vieira de; MEDEIROS, Josimar dos Santos. Caracterização Epidemiológica da Leishmaniose Visceral Humana no Nordeste Brasileiro entre 2010 e 2017. Journal of Biology \& Pharmacy and Agricultural Management. v. 14, n. 4, out/dez 2018. http://revista.uepb.edu.br/index.php/biofarm/article/view/4475/2588.

MACHADO, Claudia.; CASTRO, Bruno B. Relações hospedeiro-parasita. Revista de Ciência Elementar. v. 7, n. 4, p. 1-5, dez. 2019.

MARCELINO, Regiana Lucia. et al. Parasitoses de veiculação hídrica em águas urbanas. ANALECTA. v. 4, n. 4, p. 179-194, nov. 2018.

MICHALICK, Marilene Susan Marques; RIBEIRO, R. R.; SILVA, S. M. Gênero Leishmania. In: NEVES, David Pereira. Parasitologia Humana. 13. ed. São Paulo: Atheneu, Cap 7, p. 4147, 2016.

ORTIZ, Rafael Carneiro; ANVERSA, Laís. Epidemiologia da Leishmaniose visceral em Bauru, São Paulo, no período de 2004 a 2012: um estudo descritivo. Epidemiol Serv Saúde. v. 24, n. 1, p:97-104, 2015.

PINTO, Eduardo Ribeiro; NEPOMUCENO, Erivelton Geraldo; CAMPANHARO, Adriana Susana Lopes de Oliveira. O impacto da topologia de redes complexas no controle da propagação de doenças infecciosas. Proceeding Series of the Brazilian Society of Computational and Applied Mathematics v. 7, n. 1, p. 1-2, 2020.

ROCHA, M.A.N. et al. Aspectos epidemiológicos da leishmaniose visceral humana e canina no Estado de Alagoas, Nordeste, Brasil. Braz. J. Biol. v. 78, n. 4, p : 609-614, 2018.

SEGURADO, Aluisio Cotrim; CASSENOTE, Alex Jones; LUNA, Expedito de Albuquerque. Saúde nas metrópoles-Doenças infecciosas. Estudos avançados. v. 30, n.86, p. 29-49, jan/abr. 2016.

SOUSA, Jaqueline Maria dos Santos; RAMALHO, Walter Massa; MELO, Marcia Almeida de. Demographic and clinical characterization of human visceral leishmaniasis in the State of Pernambuco, Brazil, between 2006 and 2015. Rev Soc Bras Med Trop. v. 51, n. 5, Set-Out, 2018.

WALDMAN, Eliseu Alves; SATO, Ana Paula Sayuri. Trajetória das doenças infecciosas no Brasil nos últimos 50 anos: um contínuo desafio. Revista de Saúde Pública. v. 50, n. 68, p. 1-18, set. 2016.

WHO - World Health Organization (Organização Mundial da Saúde). Leishmaniasis 2020a. Disponível em: https://www.who.int/news-room/fact-sheets/detail/leishmaniasis.

WHO - World Health Organization (Organização Mundial da Saúde). Leishmaniasis, diagnosis, detection and surveillance 2020b. Disponível em:

https://www.who.int/leishmaniasis/surveillance/en/. 